\title{
Necitumumab plus Gemcitabine and Cisplatin as First-Line Therapy in Patients with Stage IV EGFR- Expressing Squamous Non-Small-Cell Lung Cancer: German Subgroup Data from an Open-Label, Randomized Controlled Phase 3 Study (SQUIRE)
}

\author{
Martin Reck $^{a}$ Michael Thomas $^{b}$ Cornelia Kropf-Sanchen ${ }^{c}$ Jörg Mezger ${ }^{d}$ \\ Mark A. Socinski ${ }^{\mathrm{H}}$ Henrik Depenbrock ${ }^{f}$ Victoria Soldatenkova $^{\mathrm{g}}$ Jacqueline Brown $^{\mathrm{h}}$ \\ Thomas Krause $^{f}$ Nick Thatcher ${ }^{i}$ \\ ${ }^{a}$ Department of Thoracic Oncology, Airway Research Center North (ARCN), German Center for Lung Research (DZL), \\ German Center for Lung Research (DZL), LungenClinic Grosshansdorf, Grosshansdorf, Germany; \\ ${ }^{b}$ Internistische Onkologie der Thoraxtumoren, Thoraxklinik im Universitätsklinikum Heidelberg, \\ Translational Lung Research Center Heidelberg (TLRC-H), Heidelberg, Germany; \\ ${ }^{c}$ Department of Internal Medicine II, University Hospital of UIm, UIm, Germany; \\ ${ }^{\mathrm{d}}$ Medical Department II, St. Vincentius Hospitals, Karlsruhe, Germany; \\ e Division of Hematology/Oncology University of Pittsburgh, Pittsburgh, PA, USA; \\ ${ }^{f}$ Medical Department, Oncology, Lilly Deutschland GmbH, Bad Homburg, Germany; \\ gEuropean Statistics, Lilly Deutschland GmbH, Bad Homburg, Germany; \\ hEli Lilly and Company, Erl Wood Manor, Windlesham, UK; \\ i The Christie Hospital, Manchester, UK
}

\section{Keywords}

Necitumumab - Squamous NSCLC - SQUIRE .

German subgroup data - EGFR-expressing tumors

\section{Summary}

Background: In the SQUIRE study, adding the antiepidermal growth factor receptor (EGFR) IgG1 antibody necitumumab to first-line gemcitabine and cisplatin $(\mathrm{GC}+\mathrm{N})$ in advanced squamous non-small-cell lung cancer (sqNSCLC) significantly improved overall survival (OS); the safety profile was acceptable. We explored data for the German subpopulation ( $N=96$ ) of SQUIRE patients with EGFR-expressing tumors. Patient and Methods: Patients with stage IV sqNSCLC were randomized 1:1 to up to 6 cycles of open-label GC $+\mathrm{N}$ or GC alone. $\mathrm{GC}+\mathrm{N}$ patients with no progression continued on necitumumab monotherapy until disease progression or intolerable toxicity. The primary endpoint was OS; the

Martin Reck and Michael Thomas contributed equally to this manuscript. secondary endpoints included progression-free survival (PFS), safety and health-related quality of life (EQ-5D, Lung Cancer Symptom Scale (LCSS)). Results: The 96 German SQUIRE patients with EGFR-expressing tumors ( $G C+N 42, G C 54)$ received a median of 4 GC cycles; the $\mathrm{GC}+\mathrm{N}$ patients received 5 cycles of necitumumab. Adding necitumumab was associated with $41 \%$ risk reduction of death (hazard ratio (HR) $0.59,95 \%$ confidence interval (Cl) $0.37-0.94, p=0.026$ ) and $44 \%$ risk reduction of progression (HR 0.56, 95\% $\mathrm{Cl} 0.33-0.95, \mathrm{p}=0.029$ ). Adverse events typically associated with EGFR antibody treatment (including rash, hypomagnesemia) were more common with $\mathrm{GC}+\mathrm{N}$. The time to deterioration of the EQ-5D and LCSS scores showed no notable differences between the treatment arms, except for appetite loss (delayed for $\mathrm{GC}+\mathrm{N}$ ). Conclusion: The survival benefit from adding necitumumab to first-line GC was more pronounced in the German SQUIRE subpopulation with EGFR-expressing tumors than in the overall (intentionto-treat) population; toxicity was manageable and consistent with the overall population.

(c) 2016 S. Karger GmbH, Freiburg

\begin{tabular}{|c|c|}
\hline KARGER & $\begin{array}{l}\text { (c) } 2016 \text { S. Karger GmbH, Freiburg } \\
1016-6262 / 16 / 0396-000539 \$ 39.50 / 0\end{array}$ \\
\hline $\begin{array}{l}\text { Fax +4976145207 } 14 \\
\text { Information@Karger.com } \\
\text { www.karger.com }\end{array}$ & $\begin{array}{l}\text { This article is licensed under the Creative Commons Attribution-Non- } \\
\text { Commercial-NoDerivatives } 4.0 \text { International License (CC BY-NC-ND) } \\
\text { (htttp://www.karger.com/Services/OpenAccessLicense). Usage and } \\
\text { distribution for commercial purposes as well as any distribution of }\end{array}$ \\
\hline
\end{tabular}




\section{Introduction}

Platinum-based systemic chemotherapy has been the standard of care for unresectable, metastatic non-small-cell lung cancer (NSCLC) for decades and is the recommended first-line treatment for most patients with good performance status (PS 0-2) [1]. Recent advances have resulted from the addition of targeted anticancer drugs to chemotherapy and from the availability of pemetrexed for NSCLC of non-squamous histology. Targeted agents aim to inhibit tumor growth by interfering with cell signaling involved in tumor progression, e.g. by blocking the signal transduction through the epidermal growth factor receptor (EGFR). However, the use of EGFR tyrosine kinase inhibitors in NSCLC has been confined to patients with non-squamous histology - primarily adenocarcinoma - with an activating EGFR mutation [2]. Squamous cell NSCLCs (sqNSCLCs), which make up 30\% of all NSCLCs [3], are different. They rarely carry activating EGFR mutations [4], and initial trials evaluating EGFR tyrosine kinase inhibitors in patients with sqNSCLCs were not successful. Development plans for several agents were halted due to their toxicity profiles [3]. One exception was the phase 3 FLEX trial where the addition of the chimeric EGFR antibody cetuximab to first-line treatment with vinorelbine and cisplatin improved the overall survival (OS) of patients with EGFR protein-expressing, advanced NSCLC of any histological subtype [5], in particular in the subgroup of squamous cell cancers. As expected with an anti-EGFR antibody, grade 3 acne-like skin rash $(10 \%$ vs. $<1 \%)$, grade $3 / 4$ diarrhea (5\% vs. $2 \%$ ), and grade $3 / 4$ infusion-related reactions ( $3 \%$ vs. $1 \%)$ were more common in the cetuximab arm [5]. The proportion of patients who had grade $3 / 4$ febrile neutropenia was also higher in the cetuximab arm (22\% vs. $15 \%)$ [5].

The recently published multinational 'Squamous NSCLC Treatment with the Inhibitor of EGF Receptor' (SQUIRE) trial was the largest randomized trial so far specifically evaluating a targeted agent, necitumumab, in patients with squamous cell cancer [6]. Necitumumab is a DNA-derived second-generation human immunoglobulin G1 (IgG1) monoclonal antibody that specifically blocks the EGFR ligand binding site of the EGFR. The addition of necitumumab to gemcitabine and cisplatin doublet $(\mathrm{GC}+\mathrm{N})$ chemotherapy was associated with a $16 \%$ reduction in the risk of death [6]. The typical EGFR-associated toxicities were observed in the GC + $\mathrm{N}$ arm, but there was no aggravation of chemotherapy-associated toxicities; the overall safety profile was considered acceptable. Also, safety profiles were generally similar for younger $(<70$ years) and older ( $\geq 70$ years) patients [6].

SQUIRE had a total population of more than 1,000 patients [6]. The large sample size opens up the opportunity to explore the treatment effects in specific subsets of patients, such as patients with EGFR-expressing tumors or country-specific subgroups, to identify any subgroups of patients with an additional benefit. Patients from different countries might differ in disease characteristics or comorbidities, post-study treatment, or the routine clinical practice used, in particular if healthcare systems are different. Therefore, we explored the benefit of adding necitumumab to GC treatment specifically in the subset of SQUIRE patients enrolled in Germany, and then extended this exploration to a larger subset of patients enrolled in Western European countries. Tissue collection for study participants was mandatory, and knowing that necitumumab is targeting the EGFR, we pre-specified efficacy analyses for the subpopulation of SQUIRE patients with EGFR-expressing tumors [7].

\section{Methods}

Study Design

SQUIRE (NCT00981058) was an open-label, multicenter, 2-arm, randomized phase 3 study, to evaluate the effects of adding necitumumab to firstline GC chemotherapy in patients with previously untreated metastatic sqNSCLC [6]. The study was designed as open-label study, based on the assumption that neither the physicians nor the patients would remain blinded due to the expected occurrence of acne-like rash in the GC $+\mathrm{N}$ treatment, a class effect of EGFR antibodies.

SQUIRE included mandatory pretreatment tissue collection. Tumor EGFR protein expression was assessed by immunohistochemistry (IHC) in a central laboratory with the EGFR PharmDx Kit (Dako, Glostrup, Denmark) and evaluated independently by 2 pathologists.

The study was done in compliance with the Declaration of Helsinki, the International Conference on Harmonisation Guidelines for Good Clinical Practice, and applicable local regulations. The protocol was approved by the ethics committees of all participating centers, and all patients provided written informed consent before study entry. An independent data monitoring board assessed treatment safety during the study on a regular basis. The detailed study design and randomization procedures have been published in the main SQUIRE manuscript [6].

Patients

Between January 2010 and February 2012, a total of 1,093 patients were enrolled at 184 sites in 26 different countries, including 20 sites throughout Germany. Here, we report results for a subset of 96 patients with EGFRexpressing tumors who were enrolled in Germany, and additionally present supporting data for a larger population of 300 patients with EGFR-expressing tumors enrolled in 9 Western European countries (Germany, France, Spain, Greece, Italy, United Kingdom (UK), Portugal, Austria, and Belgium). Adults ( $\geq 18$ years) with histologically or cytologically confirmed stage IV sqNSCLC (according to the American Joint Committee on Cancer (AJCC) Cancer Staging Manual, 7th edition) were eligible for enrolment. Other key inclusion criteria were an Eastern Cooperative Oncology Group (ECOG)-PS of 0-2, adequate organ function, and the availability of tumor tissue for biomarker analysis. Key exclusion criteria were: previous chemotherapy for advanced NSCLC, major surgery in the 4 weeks before randomization, chest irradiation within the 12 weeks before randomization, the presence of brain metastases that were symptomatic or needed ongoing treatment with steroids or anticonvulsants, clinically relevant coronary artery disease or uncontrolled congestive heart failure, and grade $\geq 2$ peripheral neuropathy (National Cancer Institute-Common Terminology Criteria for Adverse Events (NCI-CTCAE) version 3.0). Detailed inclusion and exclusion criteria have been published in the main SQUIRE manuscript [6].

\section{Treatment}

Patients were randomized 1:1 to receive a maximum of 63 -week cycles of open-label treatment with GC or GC + N. Gemcitabine $1,250 \mathrm{mg} / \mathrm{m}^{2}$ was administered intravenously over $30 \mathrm{~min}$ on days 1 and 8 of each cycle. Cisplatin $75 \mathrm{mg} / \mathrm{m}^{2}$ was administered intravenously over $120 \mathrm{~min}$ on day 1 of each cycle, starting at least $30 \mathrm{~min}$ after the gemcitabine administration. Patients assigned to $\mathrm{GC}+\mathrm{N}$ received necitumumab at an absolute dose of $800 \mathrm{mg}$ intravenously 


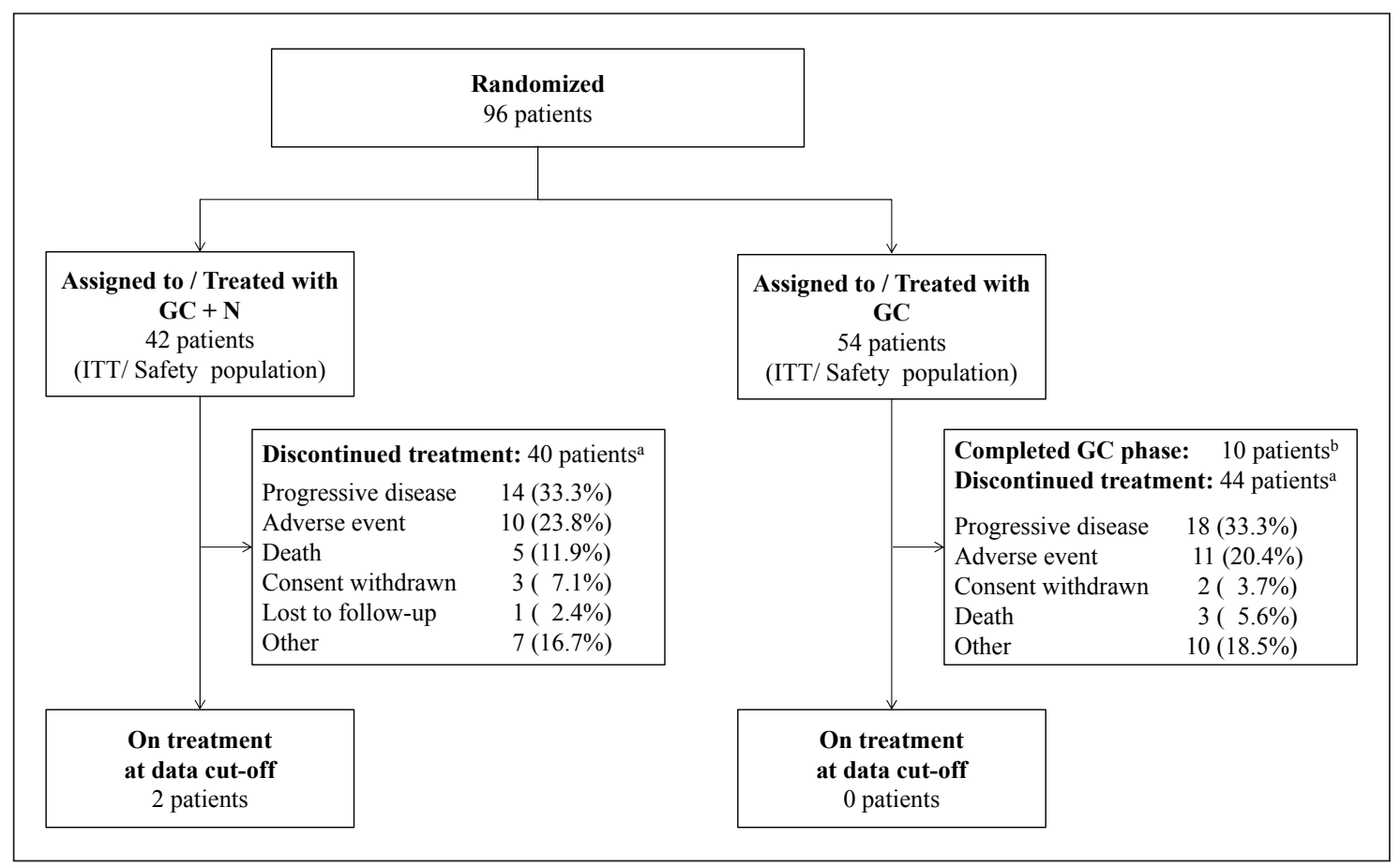

Fig. 1. Disposition of patients with EGFR-expressing tumors enrolled in Germany $(\mathrm{N}=96)$.

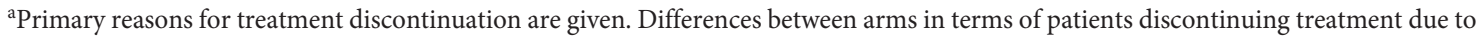
disease progression and withdrawal of consent are attributable to the study design; i.e., patients in the GC + N arm could receive up to 6 cycles of GC chemotherapy plus necitumumab followed by necitumumab continuation therapy until withdrawal criteria were met, whereas patients in the GC arm could only receive up to 6 cycles of GC chemotherapy treatment and were then followed for disease progression.

bPatients who completed 6 cycles of GC chemotherapy. GC = gemcitabine plus cisplatin, EGFR = epidermal growth factor receptor, $\mathrm{GC}+\mathrm{N}=$ gemcitabine plus cisplatin plus necitumumab.

on days 1 and 8, over a minimum of $50 \mathrm{~min}$ and before gemcitabine administration. Antiemetic premedication for gemcitabine and cisplatin was administered according to local practice. Pre-emptive treatment for skin toxicity was allowed only after the first cycle. Hypomagnesemia was treated according to local practice. After 6 cycles of chemotherapy, GC $+\mathrm{N}$ patients who had not progressed continued on necitumumab alone until radiographic documentation of progressive disease (PD) or intolerable toxicity (continuation phase). Dose modifications of chemotherapy or necitumumab were allowed according to protocoldefined criteria [6].

\section{Outcomes}

The primary outcome of the study was OS, defined as the time from randomization to death from any cause. Secondary endpoints reported here include progression-free survival (PFS), objective tumor response, health-related quality of life (HRQoL), and safety of necitumumab.

Tumor response was assessed radiographically according to Response Evaluation Criteria in Solid Tumors (RECIST) 1.0 [8] at baseline (i.e. within 21 days before randomization) and then every 6 weeks following the first dose of study therapy until radiographic documentation of $\mathrm{PD}$. The objective response rate was defined as the proportion of patients with a best overall response of complete response (CR) or partial response (PR). PFS was defined as the time from randomization until the first radiographic documentation of objective $\mathrm{PD}$ or death from any cause. Adverse events were coded according to the Medical Dictionary for Regulatory Activities (MedDRA, version 16.0) and graded using NCI-CTCAE version 3.0. HRQoL was assessed using the EuroQol five dimensions questionnaire (EQ-5D) [9] and the Lung Cancer Symptom Scale (LCSS) [10]. The EQ-5D captures the patient-reported health status and was converted to a single index value using the published algorithm for the UK general population, in the absence of a validated algorithm for the German general popula- tion. Secondly, SQUIRE patients rated their own health state on a $100-\mathrm{mm}$ visual analogue scale (VAS). The LCSS evaluates patient-reported lung cancer symptoms as well as the patient's ability to carry out normal activities and the current quality of life. The time to deterioration of the EQ-5D VAS, EQ-5D index and LCSS scores was calculated as the time from randomization to the first post-baseline deterioration. For the EQ-5D VAS score, deterioration was defined as an increase of $\geq 12$ points from the baseline score [11]. For the EQ-5D index score, deterioration was defined as an increase of $\geq 0.11$ points from the baseline score [11]. For the LCSS scores, deterioration was defined as an increase of $\geq 15 \mathrm{~mm}$ from the baseline score [12].

\section{Statistical Analysis}

The planned total sample size for SQUIRE was 1,080 patients. The primary analysis was planned to be conducted as soon as 844 deaths had occurred [6]. Exploratory analyses were pre-specified for patients with EGFR protein-expressing $($ EGFR $>0)$ and -non-expressing $($ EGFR $=0)$ tumors. Here, we report exploratory subgroup data for the subset of patients with EGFR-expressing tumors enrolled in Germany $(\mathrm{N}=96)$, and for patients with EGFR-expressing tumors enrolled in the 9 Western European countries $(\mathrm{N}=300)$. Data cut-off was on the same date as for the primary analysis (June 17, 2013). All randomized patients were included in the efficacy analysis. Safety was assessed in all patients who received at least 1 dose of study medication.

OS, PFS, and the time to deterioration of the EQ-5D and LCSS scores were estimated using the Kaplan-Meier method [13] and compared between treatment arms by unstratified log-rank tests. Hazard ratios (HRs) and their $95 \%$ confidence intervals (CIs) for GC $+\mathrm{N}$ versus GC were estimated from unstratified Cox proportional hazards models [14]. The objective response rate was estimated by treatment arm. SAS version 9.1.3 was used for all data analyses. 
Table 1. Baseline characteristics of patients with EGFR-expressing tumors enrolled in Germany ( $\mathrm{N}=96)$

\begin{tabular}{|c|c|c|}
\hline & $\begin{array}{l}\mathrm{GC}+\mathrm{N} \text { arm } \\
(\mathrm{N}=42)\end{array}$ & $\begin{array}{l}\text { GC arm } \\
(\mathrm{N}=54)\end{array}$ \\
\hline Median age, years (range) & $64(51-74)$ & $63.5(47-75)$ \\
\hline \multicolumn{3}{|l|}{ Age group, n (\%) } \\
\hline$<65$ years & $23(54.8)$ & $30(55.6)$ \\
\hline$\geq 65$ years & $19(45.2)$ & $24(44.4)$ \\
\hline$<70$ years & $36(85.7)$ & $45(83.3)$ \\
\hline$\geq 70$ years & $6(14.3)$ & $9(16.7)$ \\
\hline Sex, $\mathrm{n}(\%)$ male & $31(73.8)$ & $41(75.9)$ \\
\hline Ethnic origin, $\mathrm{n}(\%)$ white & $42(100)$ & $52(96.3)$ \\
\hline Smoking history, $\mathrm{n}(\%)$ current smoker & $40(95.2)$ & $52(96.3)$ \\
\hline \multicolumn{3}{|l|}{ ECOG-PS, n (\%) } \\
\hline $0-1$ & $42(100)$ & $51(94.4)$ \\
\hline 2 & 0 & $3(5.6)$ \\
\hline \multicolumn{3}{|l|}{ Disease histology, n (\%) } \\
\hline Squamous & $42(100)$ & $54(100)$ \\
\hline \multicolumn{3}{|l|}{ Disease stage, $\mathrm{n}(\%)$} \\
\hline IV & $41(97.6)$ & $53(98.1)$ \\
\hline IIIB (without malignant pleural effusion) ${ }^{\mathrm{a}}$ & $1(2.4)$ & $1(1.9)$ \\
\hline \multicolumn{3}{|c|}{ Number of metastatic organ systems, $n(\%)$} \\
\hline 1 & $9(21.4)$ & $5(9.3)$ \\
\hline 2 & $13(31.0)$ & $19(35.2)$ \\
\hline$>2$ & $20(47.6)$ & $30(55.6)$ \\
\hline \multicolumn{3}{|l|}{ Most common sites of metastatic disease, $\mathrm{n}(\%)$} \\
\hline Lung & $34(81.0)$ & $44(81.5)$ \\
\hline Lymph nodes & $27(64.3)$ & $41(75.9)$ \\
\hline Bone & $9(21.4)$ & $21(38.9)$ \\
\hline Pleural & $8(19.0)$ & $16(29.6)$ \\
\hline Liver & $9(21.4)$ & $7(13.0)$ \\
\hline Brain & $5(11.9)$ & $1(1.9)$ \\
\hline \multicolumn{3}{|c|}{$\begin{array}{l}\text { aThe patients with stage IIIB disease without malignant pleural effusion were } \\
\text { accidentally enrolled. } \\
\text { AJCC = American Joint Committee on Cancer, ECOG-PS = Eastern } \\
\text { Cooperative Oncology Group performance status, EGFR = epidermal growth } \\
\text { factor receptor, GC = gemcitabine plus cisplatin, GC + N = gemcitabine } \\
\text { plus cisplatin plus necitumumab, N = number of patients per treatment arm, } \\
n=\text { number of patients with event. }\end{array}$} \\
\hline
\end{tabular}

\section{Results}

\section{Patient Disposition}

Of the 1,093 patients enrolled into SQUIRE, 108 were enrolled in Germany. Of these, 97 patients had tumor tissue available and 96 with EGFR-expressing tumors were included in the analysis. Of these, 42 patients were randomly assigned to GC $+\mathrm{N}$ and 54 to GC and entered the chemotherapy phase (fig. 1). $\mathrm{PD} /$ death (GC $+\mathrm{N}$ $45.2 \%$, GC 38.9\%) and adverse events (GC + N 23.8\%, GC 20.4\%) were the most common reasons for treatment discontinuation in the German subpopulation (fig. 1). Differences between arms in terms of patients discontinuing treatment due to disease progression were attributable to the study design: patients in the GC $+\mathrm{N}$ arm received up to 6 treatment cycles with GC plus necitumumab during the chemotherapy phase followed by necitumumab continuation treatment until withdrawal criteria were met, whereas pa- tients in the GC arm received up to 6 cycles of GC chemotherapy. In the $\mathrm{GC}+\mathrm{N}$ arm, 13 of 42 patients (31.0\%) continued with necitumumab monotherapy after completing the chemotherapy phase. 2 of these patients were still receiving necitumumab at the data cutoff date.

The larger population of SQUIRE patients enrolled in the 9 Western European countries, including Germany, had a total of 348 patients. Of these, 313 had tumor tissue available and 300 with EGFR-expressing tumors were included in the analysis (GC + N 145, GC 155 patients; 298 evaluable for safety). Data for this population are presented in appendix 2,www.karger.com/?DOI=448085.

\section{Patient Characteristics}

The demographic and disease characteristics of the German subpopulation with EGFR-expressing tumors were generally similar to those for the overall study population, except for the location of metastases. In particular, bone metastases were more frequent in the German subpopulation than in the overall population $(31.3 \%$ vs. 23.0\%; appendix table S1.1, www.karger.com/?DOI=448085). The baseline characteristics (age, ethnic origin, sex, PS, tumor stage) of the German subpopulation were similar in both treatment arms $(75.0 \%$ male, $15.6 \% \geq 70$ years; table 1$)$, except for the locations of metastases which were distributed differently. In particular, liver and brain metastases were more frequent in the $\mathrm{GC}+\mathrm{N}$ arm whereas bone and pleural metastases were more common in the GC arm (table 1). This imbalance was less pronounced in the larger Western European population (appendix table S2.1, www. karger.com/?DOI=448085).

\section{Treatment Exposure}

Patients of the German subpopulation received a median of 4 GC treatment cycles in both arms (appendix table S1.2, www. karger.com/?DOI=448085). For gemcitabine/cisplatin, the median relative dose intensities were $81.8 \% / 95.6 \%$ in the GC $+\mathrm{N}$ arm and $85.6 \% / 91.3 \%$ in the GC arm. In the GC + N arm, patients received a median of 5 cycles of necitumumab overall. The 13 patients who started the necitumumab continuation phase received a median of 3 cycles (maximum 41 cycles) of single-agent necitumumab after completing the chemotherapy phase (appendix table S1.2, www. karger.com/?DOI=448085). The median relative dose intensity for necitumumab was $92.6 \%$ overall and $95.5 \%$ during the continuation phase.

\section{Survival and Response Data}

Key efficacy data for the German subpopulation are summarized in table 2. The addition of necitumumab to GC chemotherapy was associated with a $41 \%$ reduction in the risk of death (HR: 0.59, 95\% CI $0.37-0.94, \mathrm{p}=0.026$ ). The median OS was 12.4 months in the GC $+\mathrm{N}$ arm compared with 8.4 months in the GC arm. The KaplanMeier plots (fig. 2a) showed an early separation of the OS curves in favor of the GC + N arm; this separation was maintained during the whole duration of the study. The median duration of follow-up at data cut-off was 30.3 months in the GC $+\mathrm{N}$ and 36.5 months in the $\mathrm{GC}$ arm ( $\mathrm{p}=0.403)$; the censoring rate was $20.8 \%$. 
Table 2. Efficacy endpoints of patients with EGFR-expressing tumors enrolled in Germany $(\mathrm{N}=96)$
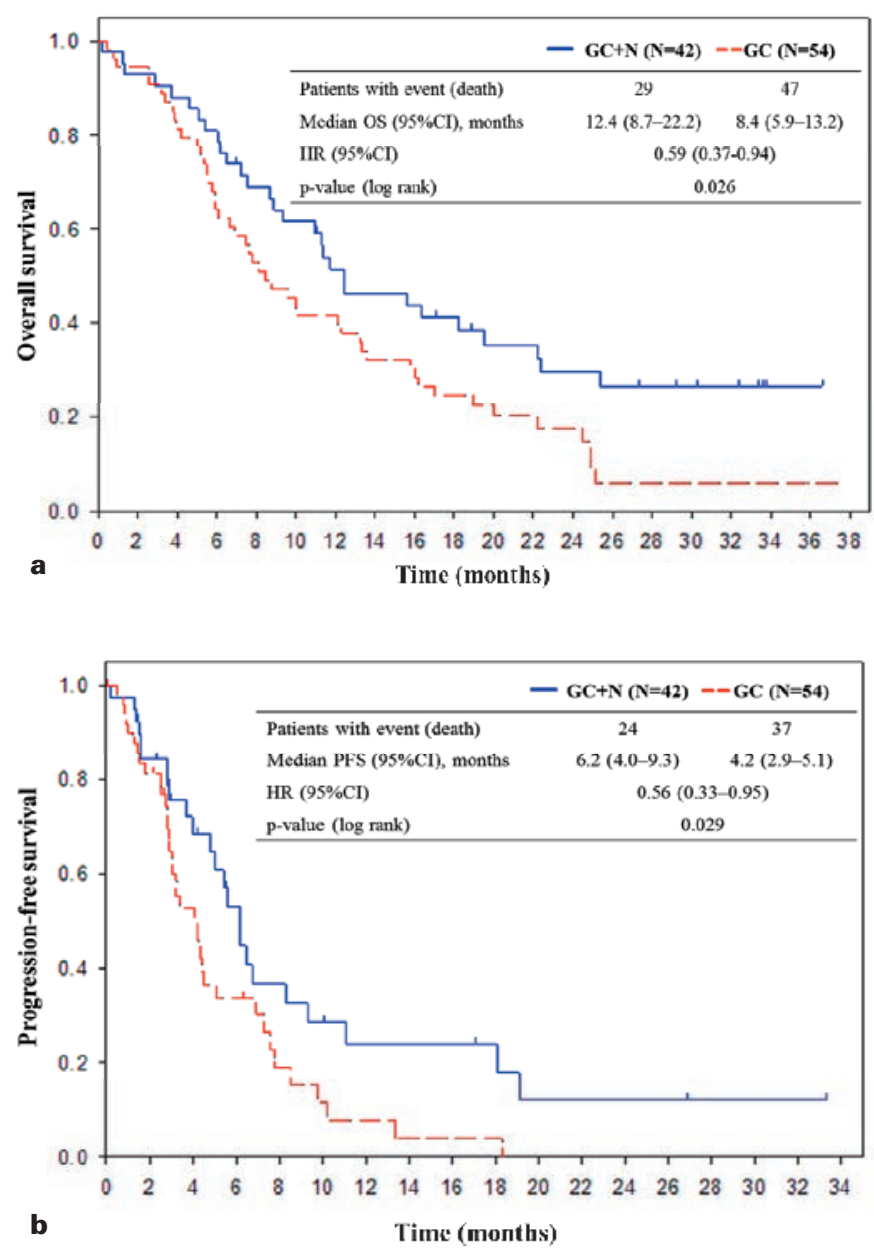

Fig. 2. Overall (a) and progression-free (b) survival of patients with EGFRexpressing tumors enrolled in Germany $(\mathrm{N}=96)$, Kaplan-Meier plots.
$\mathrm{GC}+\mathrm{N} \operatorname{arm}(\mathrm{N}=42) \quad \mathrm{GC} \operatorname{arm}(\mathrm{N}=54)$

OS

HR (95\% CI)

p-Value

$0.59(0.37-0.94)$

0.026

$29(69.0) \quad 47(87.0)$

$12.4(8.7-22.2) \quad 8.4(5.9-13.2)$

PFS

HR (95\% CI)

p-Value

Events, $\mathrm{n}(\%)$

Median PFS, months (95\% CI)

Best overall response, $\mathrm{n}(\%)$

CR

PR

Objective response rate (CR + PR), \% (95\% CI)

$\mathrm{SD}$

Disease control rate $(\mathrm{CR}+\mathrm{PR}+\mathrm{SD}), \%(95 \% \mathrm{CI})$

Not evaluable/not assessed combined

$\mathrm{CI}=$ Confidence interval, $\mathrm{CR}=$ complete response, $\mathrm{EGFR}=$ epidermal growth factor receptor $\mathrm{GC}=$ gemcitabine plus cisplatin, $\mathrm{GC}+\mathrm{N}$, gemcitabine plus cisplatin plus necitumumab, $\mathrm{HR}=$ hazard ratio, $\mathrm{N}$ = number of patients per treatment arm, $\mathrm{n}=$ number of patients with event, $\mathrm{OS}=$ overall survival, $\mathrm{PFS}=$ progression-free survival, $\mathrm{PD}=$ progressive disease, $\mathrm{PR}=$ partial response, $\mathrm{SD}=$ stable disease.

$\begin{array}{lc} & 0.56(0.33-0.95) \\ & 0.029 \\ 24(57.1) & 37(68.5) \\ 6.2(4.0-9.3) & 4.2(2.9-5.1) \\ & \\ 0 & 0 \\ 16(38.1) & 12(22.2) \\ 38.1(25.0-53.2) & 22.2(13.2-34.9) \\ 18(42.9) & 27(50.0) \\ 81.0(66.7-90.0) & 72.2(59.1-82.4) \\ 4(9.5) & 6(11.1) \\ 4(9.5) & 9(16.7)\end{array}$


Table 4. Summary of adverse events during the chemotherapy phase in patients with EGFR-expressing tumors enrolled in Germany $(\mathrm{N}=96)$

\begin{tabular}{|c|c|c|c|c|}
\hline & \multicolumn{2}{|c|}{$\mathrm{GC}+\mathrm{N} \operatorname{arm}(\mathrm{N}=42)$} & \multicolumn{2}{|c|}{$\mathrm{GC} \operatorname{arm}(\mathrm{N}=54)$} \\
\hline & Any grade & Grade $\geq 3$ & Any grade & Grade $\geq 3$ \\
\hline Any adverse event ${ }^{\mathrm{a}}$ & $42(100)$ & $33(78.6)$ & $54(100)$ & $40(74.1)$ \\
\hline Serious & $25(59.5)$ & & $31(57.4)$ & \\
\hline Leading to treatment discontinuation & $17(40.5)$ & $12(28.6)$ & $13(24.1)$ & $8(14.8)$ \\
\hline Leading to death ${ }^{\mathrm{b}}$ & $4(9.5)$ & & $4(7.4)$ & \\
\hline \multicolumn{5}{|l|}{ Hematologic toxicities $^{c}, \mathrm{n}(\%)$} \\
\hline Neutropenia & $14(33.3)$ & $11(26.2)$ & $21(38.9)$ & $19(35.2)$ \\
\hline Febrile neutropenia & 0 & 0 & 0 & 0 \\
\hline Anemia & $9(21.4)$ & $4(9.5)$ & $22(40.7)$ & $9(16.7)$ \\
\hline Thrombocytopenia & $14(33.3)$ & $8(19.0)$ & $17(31.5)$ & $6(11.1)$ \\
\hline \multicolumn{5}{|l|}{ Non-hematologic toxicities of special interest ${ }^{c}, \mathrm{n}(\%)$} \\
\hline Fatigue & $24(57.1)$ & $2(4.8)$ & $32(59.3)$ & $8(14.8)$ \\
\hline Hypomagnesemia & $8(19.0)$ & $3(7.1)$ & $5(9.3)$ & $3(5.6)$ \\
\hline Skin reactions & $35(83.3)$ & $3(7.1)$ & $11(20.4)$ & 0 \\
\hline Rash & $33(78.6)$ & $2(4.8)$ & $10(18.5)$ & 0 \\
\hline Hypersensitivity/infusion-related reactions & $4(9.5)$ & $1(2.4)$ & $1(1.9)$ & 0 \\
\hline Conjunctivitis & $6(14.3)$ & 0 & $5(9.3)$ & 0 \\
\hline Interstitial lung disease & 0 & 0 & 0 & 0 \\
\hline Arterial thromboembolic events & $3(7.1)$ & $2(4.8)$ & $3(5.6)$ & $1(1.9)$ \\
\hline Venous thromboembolic events & $10(23.8)$ & $7(16.7)$ & $6(11.1)$ & $3(5.6)$ \\
\hline Vomiting & $8(19.0)$ & 0 & $10(18.5)$ & $2(3.7)$ \\
\hline
\end{tabular}

${ }^{\text {a}}$ For the GC $+\mathrm{N}$ arm, this includes all adverse events starting up to 30 days after the last dose of GC chemotherapy. Adverse events starting later during the necitumumab continuation phase were evaluated separately (adverse events during necitumumab continuation phase; appendix table S1.3).

${ }^{\mathrm{b}}$ Excluding deaths due to disease progression.

${ }^{c}$ Hematologic adverse events and non-hematologic adverse events of special interest include preferred terms and related terms as identified by the authoring team (except for febrile neutropenia and weight loss, which are only preferred terms).

$\mathrm{GC}=$ Gemcitabine plus cisplatin, EGFR = epidermal growth factor receptor, $\mathrm{GC}+\mathrm{N}=$ gemcitabine plus cisplatin plus necitumumab, $\mathrm{N}=$ number of patients per treatment $\mathrm{arm}, \mathrm{n}=$ number of patients with event.

\section{Post-Study Systemic Therapy}

In both treatment arms, $\geq 50 \%$ of the patients enrolled in Germany received post-study systemic therapy (GC + N 50.0\%, GC $55.6 \%)$, most commonly with docetaxel or erlotinib (table 3). Overall, post-study therapy was balanced between the treatment arms.

\section{Safety Data}

In the German subpopulation, the incidence of any treatmentemergent adverse events grade $\geq 3$ during the chemotherapy phase was $78.6 \%$ in the GC $+\mathrm{N}$ arm compared with $74.1 \%$ in the GC arm (table 4), mainly driven by an increased incidence of skin rash and hypomagnesemia in the GC $+\mathrm{N}$ arm. Treatment discontinuations due to adverse events were $40.5 \%$ with GC $+\mathrm{N}$ and $24.1 \%$ with GC. 4 patients per treatment arm had fatal adverse events not related to $\mathrm{PD}(\mathrm{GC}+\mathrm{N}$ : 1 gastric hemorrhage, 1 pneumonia, and 2 deaths from unknown cause; GC: 1 neutropenic sepsis, 1 urosepsis, 1 encephalopathy, and 1 suicide). Grade 4 toxicities were reported during the chemotherapy phase for $21.4 \%$ of the GC $+\mathrm{N}$ and $14.8 \%$ of the GC arm patients.

Skin reactions, in particular skin rash, occurred more frequently in the $\mathrm{GC}+\mathrm{N}$ than in the GC arm (table 4), but these were mainly of grade $1 / 2$. Seven patients in the GC $+\mathrm{N}$ arm (16.7\%) and 3 patients in the GC arm (5.6\%) had grade $\geq 3$ venous thromboembolic events during the chemotherapy phase. Grade 3 or higher arterial thromboembolic events were less common (GC + N 2 patients, $4.8 \%$; GC 1 patient, $1.9 \%$; table 4). No venous or arterial thromboembolic event was fatal. No case of interstitial lung disease was reported in the German subpopulation (Western European population: 2 cases in each arm).

Necitumumab continuation treatment was generally well tolerated: 4 of the 13 patients in the German subpopulation with EGFRexpressing tumors reported grade $3 / 4$ treatment-emergent adverse events during the continuation phase (no fatal events, 1 grade 4 neutropenia, 1 grade 3 anemia, 2 grade 3 skin reactions, 1 grade 3 venous thromboembolic event; appendix table S1.3, www.karger. $\mathrm{com} /$ ?DOI=448085), and no patient discontinued during the continuation phase due to adverse events. No arterial and only 1 venous thromboembolic event were reported during the continuation phase.

Patients in the $\mathrm{GC}+\mathrm{N}$ arm required fewer transfusions $(\mathrm{GC}+\mathrm{N} 26.2 \%$, GC $33.3 \%)$; there were no relevant differences regarding the proportion of patients hospitalized due to adverse events (GC + N 54.8\%, GC 51.9\%). The median duration of hospitalization due to adverse events was 12 days in the $\mathrm{GC}+\mathrm{N}$ arm and 9 days in the GC arm.

Similar safety profiles were observed in the Western European population (appendix tables S2.3 and S2.4, www.karger.com/? $D O I=448085)$ 


\section{Health-Related Quality of Life}

In both treatment arms, patients enrolled in Germany deteriorated over time in HRQoL, as assessed by EQ-5D and LCSS scores (appendix table S1.4,www.karger.com/?DOI=448085). No relevant differences in time to deterioration could be detected between the treatment arms for all but 1 (loss of appetite) of the EQ-5D and LCSS scores assessed. The median time to deterioration of the patients' appetite was substantially delayed in the GC $+\mathrm{N}$ arm, from 2.6 to 11.7 months (HR 0.47, 95\% CI 0.23-0.95).

\section{Discussion}

The overall (intent-to-treat, ITT) SQUIRE results in a broad population of more than 1,000 patients enrolled in 26 different countries showed a $16 \%$ reduction in the risk of death for patients with stage IV sqNSCLC who received the EGFR antibody necitumumab as add-on to first-line standard-of-care GC doublet chemotherapy [6] Necitumumab is known to target the EGFR. We now showed that, in the smaller subset of 96 SQUIRE patients with EGFR-expressing tumors enrolled in Germany, the survival benefit associated with the addition of necitumumab was more pronounced than for the overall population. Adding necitumumab reduced the risk of death by $41 \%$ compared with GC chemotherapy alone. This survival benefit was accompanied by a $44 \%$ reduction in the risk of progression. A similar increase in benefit was observed in the larger population of 300 patients with EGFR-expressing tumors enrolled in 9 different Western European countries (including Germany). Of note, the median OS in the GC comparator arm of the German SQUIRE and Western European populations were 8.4 and 8.9 months, respectively, and thus within the expected range of 8-10 months for patients with stage IV sqNSCLC [15], but slightly lower than the median OS for the GC arm in the SQUIRE ITT population [6].

Patients with metastatic sqNSCLC as enrolled in SQUIRE have a very high disease burden. The 2 treatment arms of the German SQUIRE subpopulation were balanced except for the distribution of the sites of metastases. Liver and brain metastases were more frequent in the GC $+\mathrm{N}$ arm whereas bone metastases were more common in the GC arm. Liver and brain metastases are associated with very poor prognosis whereas bone metastases seem to have less impact on survival $[16,17]$, indicating that the patients in the $\mathrm{GC}+\mathrm{N}$ arm might have had a poorer prognosis than the GC arm patients. In the overall population, the incidence of liver metastases was similar to that in the German subpopulation GC $+\mathrm{N}$ arm, as was the incidence of bone metastases.

There were no relevant differences regarding the median relative dose intensities of GC chemotherapy between the overall population and the German subpopulation. The median relative dose intensities of necitumumab in the $\mathrm{GC}+\mathrm{N}$ arm were also similar, as was the proportion of patients receiving post-study systemic therapy [6].

Demographic and disease characteristics as well as treatment exposure for the German and Western European populations were consistent with the larger overall population, leading us to hypothesize that other factors might explain the observed increase in benefit. The increase in benefit was highly consistent between the German and the broader Western European population, indicating the robustness of these data. Differences in ethnicity are an unlikely cause, since the majority of patients were white in both populations, German and overall [6]. Coleman et al. [18] concluded in a study from 2008 that regional differences in lung cancer survival may reflect the varying prevalence and distribution of risk factors, as well as disparities in the effective delivery of cancer control measures. In addition, a substantial proportion of the lung cancer burden across Europe may be attributed to environmental causes of cancer including social and cultural practices such as heavy smoking [18]. Further, the recently published EUROCARE-5 study also identified regional differences in lung cancer survival [19]. Cancer survival was associated with economic factors such as investment into a country's healthcare system [20]. The additional survival benefit of the German and Western European subpopulations from necitumumab might thus be associated with differences in healthcare systems and routine patient care, which might have caused imbalances, e.g., regarding the access to optimal treatment for comorbidities or the optimal post-study treatments $[21,22]$.

Consistent with safety data for the overall population [6], the addition of necitumumab to GC chemotherapy was associated with manageable toxicity, although there was an increase in adverse events leading to treatment discontinuation. Hematologic toxicity was similar in both arms; no increase in chemotherapy-associated toxicities resulted from the addition of necitumumab. Non-hematologic toxicities were as expected and included those typically associated with the anti-EGFR monoclonal antibodies $[2,5,6]$, such as thromboembolic events [23], skin reactions (rash, mainly grade $1 / 2$ ), and hypomagnesemia (mainly grade $1 / 2$ ), all of which were more common in the GC $+\mathrm{N}$ arm. Thromboembolic events have also been associated with cisplatin-based chemotherapy as well as metastatic NSCLC itself $[23,24]$. Consistently, all but 1 thromboembolic event occurred during the chemotherapy phase. There was only a single venous thromboembolic event during necitumumab continuation treatment. Hypomagnesemia occurring during necitumumab treatment can be managed if magnesium levels are routinely monitored and any decrease is treated by providing magnesium supplementation [25].

In addition to the poor prognosis, patients with advanced metastatic NSCLC suffer from a high burden of disease, caused by their cancer and their comorbidities such as fatigue, loss of appetite, dyspnea and pain, associated with a significant deterioration of HRQoL [26] and decreased OS [27]. Adding a third agent to the current standard platinum-based doublet may gain additional treatment benefit, but may also potentially be associated with negative effects on the patients' HRQoL [28]. In the German SQUIRE subpopulation, however, the addition of necitumumab to GC chemotherapy did not cause an additional impairment of health status (EQ-5D), lung cancer symptoms, the ability to carry out normal activities, and quality of life (LCSS), when compared to the GC arm. Furthermore, exploratory data from the global SQUIRE population showed that the addition of necitumumab to GC was increasingly effective as the disease burden (poor baseline LCSS) 
increased, providing the greatest survival benefit in patients with more severe symptoms or more severely reduced ability to carry out normal activities or reduced quality of life [27].

Although of high interest for clinicians and healthcare providers, the results of exploratory subgroup analyses for German patients should be interpreted with caution because the study was not powered for by-country analyses. However, the OS benefit was statistically significant in spite of the underpowered sample size, and consistent effects were seen for PFS and for both the German and Western European populations. Another limitation is that not all potentially confounding factors could be assessed, such as socioeconomic factors. Also, treatment effects observed in randomized clinical trials are not necessarily transferable to the real-life situation. Non-interventional studies evaluating the use patterns, effectiveness, and toxicity of necitumumab and other targeted agents in real-life settings would be needed to evaluate the impact of socioeconomic factors and differences in patient care.

Future studies should be specifically designed to identify which factors in routine patient care and healthcare settings might be predictive for patient benefit from necitumumab. This upcoming research, together with real-life data on the use of targeted agents, will provide more clarity about the future use of necitumumab in the routine care of patients with squamous cell histology.

\section{Conclusions}

In subsets of SQUIRE patients with EGFR-expressing tumors enrolled in Germany and other Western European countries, the addition of necitumumab to first-line GC treatment of sqNSCLC was associated with a clinically meaningful survival benefit, manageable toxicity, and no deleterious effects on HRQoL as measured by the EQ-5D and LCSS. These data substantiate the findings for the overall population indicating that the addition of necitumumab to first-line GC treatment marks the first advance to improve patient care for hard-to-treat NSCLCs of squamous histology since more than a decade.

\section{Online Supplementary Material}

Appendix 1. Additional data for patients enrolled in Germany

Appendix 2. Data for 300 patients with EGFR-expressing tumors enrolled in 9 Western European countries (including Germany)

To access the online supplementary material please refer to $w w w . k a r g e r$. $\mathrm{com} /$ ?DOI=448085.

\section{Authors' Contributions}

V.S. and T.K. were involved in the conception and design of this work. T.K. was also involved in obtaining funding. C.R., M.R., C.K.-S., J.M., A.S., and T.K. also provided administrative, technical or material support or were involved in supervision. M.R., M.T., C.K.-S., J.M., M.A.S., H.D., V.S., T.K., and N.T. were involved in the acquisition, analysis or interpretation of data. V.S. was responsible for the statistical analysis. A.S., V.S., and T.K. helped to draft the manuscript. All authors revised the manuscript critically for important intellectual content, and all authors approved the final manuscript.

\section{Acknowledgements}

This study was funded by Eli Lilly and Company. We thank the patients, their families and the study personnel across all sites for participating in this study. We thank Karin Helsberg, Trilogy Writing and Consulting GmbH, Frankfurt, Germany, for writing and editorial assistance on behalf of Eli Lilly and Company.

\section{Disclosure Statement}

M. Reck, C. Kropf-Sanchen, MA Socinski, and N. Thatcher have served as consultants (advisory board) for and have received speaker honoraria from Eli Lilly. M. Thomas received speaker honoraria from Eli Lilly, BMS, MSD; AstraZeneca, Roche, Novartis, Celgene, Pfizer, and Boehringer. H. Depenbrock, V. Soldatenkova, J. Brown, and T. Krause are Eli Lilly employees, H. Depenbrock and V. Soldatenkova also own Eli Lilly stock. J. Mezger has no conflicts of interest to disclose.

\section{References}

1 Reck M, Popat S, Reinmuth N, De Ruysscher D, Kerr KM, Peters S; ESMO Guidelines Working Group: Metastatic non-small-cell lung cancer (NSCLC): ESMO clinical practice guidelines for diagnosis, treatment and follow-up. Ann Oncol 2014;25(suppl 3):iii27-iii39.

2 Pillai RN, Ramalingam SS: Necitumumab: a new therapeutic option for squamous cell lung cancer? Transl Lung Cancer Res 2014;3:382-383.

3 Perez-Moreno P, Brambilla E, Thomas R, Soria JC: Squamous cell carcinoma of the lung: molecular subtypes and therapeutic opportunities. Clin Cancer Res 2012;18:2443-2451.

4 Rekhtman N, Paik PK, Arcila ME, Tafe LJ, Oxnard GR, Moreira AL, Travis WD, Zakowski MF, Kris MG, Ladanyi M: Clarifying the spectrum of driver oncogene mutations in biomarker-verified squamous carcinoma of lung: lack of EGFR/KRAS and presence of PIK3CA/ AKT1 mutations. Clin Cancer Res 2012;18:1167-1176
5 Pirker R, Pereira JR, Szczesna A, von Pawel J, Krzakowski M, Ramlau R, et al.: Cetuximab plus chemotherapy in patients with advanced non-small-cell lung cancer (FLEX): an open-label randomised phase III trial. Lancet 2009;373:1525-1531.

6 Thatcher N, Hirsch FR, Luft AV, Szczesna A, Ciuleanu TE, Dediu M, et al.: Necitumumab plus gemcitabine and cisplatin versus gemcitabine and cisplatin alone as first-line therapy in patients with stage IV squamous non-small-cell lung cancer (SQUIRE): an open-label, randomised, controlled phase 3 trial. Lancet Oncol 2015;16:763-774.

7 European Medicines Agency: Summary of opinion: Portrazza (necitumumab), 2015. www.ema.europa.eu/ docs/en_GB/document_library/Summary_of_opinion_-Initial_authorisation/human/003886/WC500199044. pdf [last accessed on February 10, 2015].
8 Therasse P, Arbuck SG, Eisenhauer EA, Wanders J, Kaplan RS, Rubinstein L, et al.: New guidelines to evaluate the response to treatment in solid tumors. European Organization for Research and Treatment of Cancer, National Cancer Institute of the United States, National Cancer Institute of Canada. J Natl Cancer Inst 2000;92:205-216.

9 Nord E: EuroQol: health-related quality of life measurement. Valuations of health states by the general public in Norway. Health Policy 1991;18:25-36.

10 Hollen PJ, Gralla RJ, Kris MG, Cox C, Belani CP, Grunberg SM, et al.: Measurement of quality of life in patients with lung cancer in multicenter trials of new therapies. Psychometric assessment of the Lung Cancer Symptom Scale. Cancer 1994;73:2087-2098.

11 Pickard AS, Neary MP, Cella D: Estimation of minimally important differences in EQ-5D utility and VAS scores in cancer. Health Qual Life Outcomes 2007;5:70. 
12 de Marinis F, Pereira JR, Fossella F, et al.: Lung Cancer Symptom Scale outcomes in relation to standard efficacy measures: an analysis of the phase III study of pemetrexed versus docetaxel in advanced non-small cell lung cancer. J Thorac Oncol 2008;3:30-36.

13 Kaplan EL, Meier P: Nonparametric estimation from incomplete observations. J Am Stat Assoc 1958;53 457-481.

14 Cox DR: Regression models and life-tables. J R Stat Soc Series B Stat Methodol 1972;34:187-220.

15 Scagliotti GV, De Marinis F, Rinaldi M, Crinò L Gridelli C, Ricci S, Zhao YD, Liepa AM, Peterson P, Tonato $\mathrm{M}$ : The role of histology with common first-line regimens for advanced non-small cell lung cancer: a brief report of the retrospective analysis of a three-arm randomized trial. J Thorac Oncol 2009;4:1568-1571.

16 Riihimäki M, Hemminki A, Fallah M, et al.: Metastatic sites and lung cancer. Lung Cancer 2014;86:78-84.

17 Sugiura H, Yamada K, Sugiura T, Hida T, Mitsudomi T: Predictors of survival in patients with bone metastasis of lung cancer. Clin Orthop Relat Res 2008;466: 729-736.

18 Coleman M, Alexe D, Albreht T, McKee M (eds): Responding to the Challenge of Cancer in Europe. http:// apps.who.int/iris/bitstream/10665/107879/1/E91137. pdf, 2008 [last accessed on February 15, 2015].
19 Francisci S, Minicozzi P, Pierannunzio D, Ardanaz E, Eberle A, Grimsrud TK, Knijn A, Pastorino U, Salmerón D, Trama A, Sant M; EUROCARE-5 Working Group: Survival patterns in lung and pleural cancer in Europe 1999-2007: results from the EUROCARE-5 study. Eur J Cancer 2015;51:2242-2253.

20 Baili P, Di Salvo F, Marcos-Gragera R, Siesling S, Mallone S, Santaquilani M, Micheli A, Lillini R, Francisci S; EUROCARE-5 Working Group: Age and case mixstandardised survival for all cancer patients in Europe 1999-2007: results of EUROCARE-5, a populationbased study. Eur J Cancer 2015;51:2120-2129.

21 Sant M, Minicozzi P, Primic-Žakelj M, Otter R, Francisci S, Gatta G, Berrino F, De Angelis R: Cancer survival in Europe, 2000-2007: doing better, feeling worse? Eur J Cancer 2015, doi: 10.1016/j.ejca.2015. 08.019 [Epub ahead of print]

22 Kim HS, Mitsudomi T, Soo RA, Cho BC: Personalized therapy on the horizon for squamous cell carcinoma of the lung. Lung Cancer 2013;80:249-255.

23 Petrelli E, Cabiddu M, Borgonovo K, Barni S: Risk of venous and arterial thromboembolic events associated with anti-EGFR agents: a meta-analysis of randomized clinical trials. Ann Oncol 2012;23:1672-1679.
24 Moore RA, Adel N, Riedel E, Bhutani M, Feldman DR, Tabbara NE, Soff G, Parameswaran R, Hassoun H: High incidence of thromboembolic events in patients treated with cisplatin-based chemotherapy: a large retrospective analysis. J Clin Oncol 2011;29:3466-3473.

25 Petrelli E, Borgonovo K, Cabiddu M, Ghilardi M Barni S: Risk of anti-EGFR monoclonal antibody-related hypomagnesemia: systematic review and pooled analysis of randomized studies. Expert Opin Drug Saf 2012;11(suppl 1):S9-S19.

26 Iyer S, Taylor-Stokes G, Roughley A: Symptom burden and quality of life in advanced non-small cell lung cancer patients in France and Germany. Lung Cancer 2013;2:288-293.

27 Reck M, Gralla R, Bonomi P, et al.: Maximum severity score (MSS) of baseline patient-reported Lung Cancer Symptom Scale (LCSS) as a prognostic and predictive factor for overall survival (OS) in the phase III SQUIRE study. J Clin Oncol 2015;33(suppl):abstr 8099.

28 Azim HA, Elattar I, Loeberiza FR, et al.: Third generation triplet cytotoxic chemotherapy in advanced nonsmall cell lung cancer: a systematic overview. Lung Cancer 2009;64:194-198. 